\title{
Planejamento estratégico de SI/TI: melhores práticas para educar e treinar pessoas em organizações governamentais brasileiras
}

\author{
José Gilson de Almeida Teixeira Filho \\ Mayara Benício de Barros Souza ${ }^{2}$ \\ Hermano Perrelli de Moura ${ }^{2}$
}

\begin{abstract}
Resumo: Este artigo apresenta um conjunto de melhores práticas para educar e treinar pessoas, estabelecidas pelo modelo de maturidade MMPE-SI/TI (Gov), que é direcionado ao planejamento estratégico de Sistemas de Informação/Tecnologia da Informação (SI/TI) de organizações governamentais brasileiras. Esse modelo foi concebido em conformidade com os principais modelos e normas nacionais e internacionais utilizados para definição e avaliação. $\mathrm{O}$ artigo apresenta, também, uma descrição completa do processo "Educar e treinar pessoas (ETP)", estruturado nas seguintes partes: propósito, resultados esperados, melhores práticas e produtos de trabalho. Por fim, são apresentados os resultados de uma validação realizada com 106 especialistas da área de SI/TI, que opinaram sobre a qualidade do modelo e desse processo em particular, além de ferramentas e técnicas para apoiar a sua implementação.
\end{abstract}

Palavras-chave: Melhores práticas. Educar e treinar pessoas. Planejamento estratégico de SI/TI.

\begin{abstract}
This paper presents a set of best practices to educate and train persons established by the maturity model "MMPE-SI/TI (Gov)" that is directed to strategic planning of IS/IT (Information Systems/Information Technology) brazilian governmental organizations. This model has been designed in accordance with the main models and national and international standards used for the definition and evaluation. The article also presents a complete description of the process "Educating and Training People (ETP)" which is structured into the following parts: purpose, outcomes, best practices and work products. Finally, we present the results of a validation performed with 106 experts in the field of IS/IT commented on the quality and style of this particular process, beyond of tools and techniques to support their implementation.
\end{abstract}

Keywords: Best practices. Educate and train people. Strategic planning of IS/IT.

\section{Introdução}

No contexto em que os Sistemas de Informação/Tecnologia da Informação (SI/TI) auxiliam os processos de tomada de decisões nas organizações [1], o planejamento estratégico surge como elemento vital e que deve ser construído de maneira flexível, com a participação e o comprometimento de todos os colaboradores [2].

Inicialmente, de acordo com [3], o planejamento estratégico de SI visava a explorar as perspectivas das organizações para o uso de TI, verificando os recursos necessários e desenvolvendo estratégias e planos de ação, além de ser voltado, essencialmente, para o alinhamento do desenvolvimento com as necessidades dos negócios e a busca por vantagens advindas da TI [4].

\footnotetext{
${ }^{1}$ Universidade de Pernambuco - Recife (PE) - Brasil

\{jgatf@upe.poli.br\}

${ }^{2}$ Centro de Informática, Universidade Federal de Pernambuco, Recife (PE) - Brasil.

$\{$ hermano, mbbs@cin.ufpe.br\}
}

http://dx.doi.org/10.5335/rbca.2013.2777 
Esse planejamento é o resultado da união de alguns elementos, tais como: conhecimento ao acaso, usuários, técnicas, procedimentos e influência dos SI, reconhecimento do valor dos SI (utilizados para definição da direção estratégica organizacional) e desenvolvimento de arquiteturas de informação e estratégias para aplicações, com o intuito de combinar e organizar as diversas tecnologias organizacionais [5], [6]. Dessa forma, diante de um ambiente cada vez mais influenciado pelas relações interorganizacionais dos negócios, planejar requer que as organizações lidem com diferentes fatores, prioridade e a capacidade de influência dos stakeholders [7].

Observa-se que algumas organizações elaboram seu planejamento estratégico de SI/TI, mas enfrentam grande dificuldade em avaliar a qualidade e os resultados efetivos ao longo dos anos, e, ainda, se o plano foi bem definido e coordenado. As organizações, de maneira geral, desconhecem o quanto estão aderentes às melhores práticas mundiais e dificilmente encontram formas de comparar-se (benchmarking) com outras organizações, seja no cenário nacional ou internacional [8], [9], [10], [11], [12], [13], [14], [15].

Para auxiliar as organizações a responderem a essas questões, foi elaborado o Modelo de Maturidade para Planejamento Estratégico de SI/TI (MMPE-SI/TI [Gov]), direcionado às organizações governamentais brasileiras [16]. Diante do exposto, este artigo tem como objetivo principal apresentar, em detalhes, um dos processos desse modelo, o de "Educar e treinar pessoas (ETP)", que contém um propósito, dois resultados esperados, sete melhores práticas e cinco produtos de trabalho. $\mathrm{O}$ texto também se propõe a comparar as características e funcionalidades das ferramentas e técnicas que podem ser utilizadas pela organização para a implementação desse processo.

\section{MMPE-SI/TI}

Consideram-se como melhores práticas (MPs) as visões de organizações e profissionais globais que, por meio da vivência no mercado, conseguem perceber práticas que, se utilizadas em outros contextos organizacionais, podem melhorar seu desempenho. Isso inclui a habilidade de entregar projetos de maneira previsível, consistente e com sucesso na implementação das estratégias organizacionais. Além disso, melhores práticas são dinâmicas porque evoluem com o tempo; novas e melhores abordagens são desenvolvidas para atingir o seu objetivo declarado. Utilizando-se as MPs, as probabilidades de que as metas declaradas ou os objetivos sejam alcançados aumentam consideravelmente [17], [18], [19], [20], [21].

Para [22], a competição e o dinamismo do mercado estão cada vez mais exigindo que melhores práticas mundiais sejam implantadas nas organizações. É fato que as empresas que vêm adotando essas MPs estão mais capacitadas para atingir o sucesso do que aquelas que permanecem presas a práticas superadas ou antiquadas.

O MMPE-SI/TI (Gov), nesse contexto, foi definido para auxiliar no acesso às melhores práticas mundiais e está em conformidade com os principais modelos e normas nacionais e internacionais utilizados para definição e avaliação de processos, tais como: ISO/IEC 12207, ISO/IEC 15504-1, CMMI, MPS.BR: Guia Geral, COBIT, MMGP, OPM3, PMMM [23], [24], [25], [20], [21], [26], [27], [28].

O modelo apresenta quatro áreas que auxiliam a organização a direcionar seus esforços e dar mais atenção, no momento de desenvolver o seu planejamento estratégico de SI/TI. Essas áreas foram definidas segundo a visão de diversos autores [25], [18], [29], [30], [31], [32], [33], [34], [35], [6] e recebem como entrada as melhores características dos principais modelos/metodologias/autores pesquisados por [16], além de um conjunto de melhores práticas de planejamento estratégico de SI/TI (MP.SI/TI), maturidade em gerenciamento de projetos (MP.MGP) e, especialmente, governo brasileiro (MP.GOV) [16].

Esse modelo está estruturado em três componentes, Modelo de Referência (MR), Banco de Melhores Práticas (BMP) e Método de Avaliação (MA), e apresenta quatro áreas que auxiliam na organização para direcionar seus esforços e desenvolver o seu planejamento estratégico de SI/TI: Gestão, Organização, Pessoas e Tecnologia. A Figura 1 apresenta a visão geral do MMPE-SI/TI (Gov) e de todas as suas partes. 


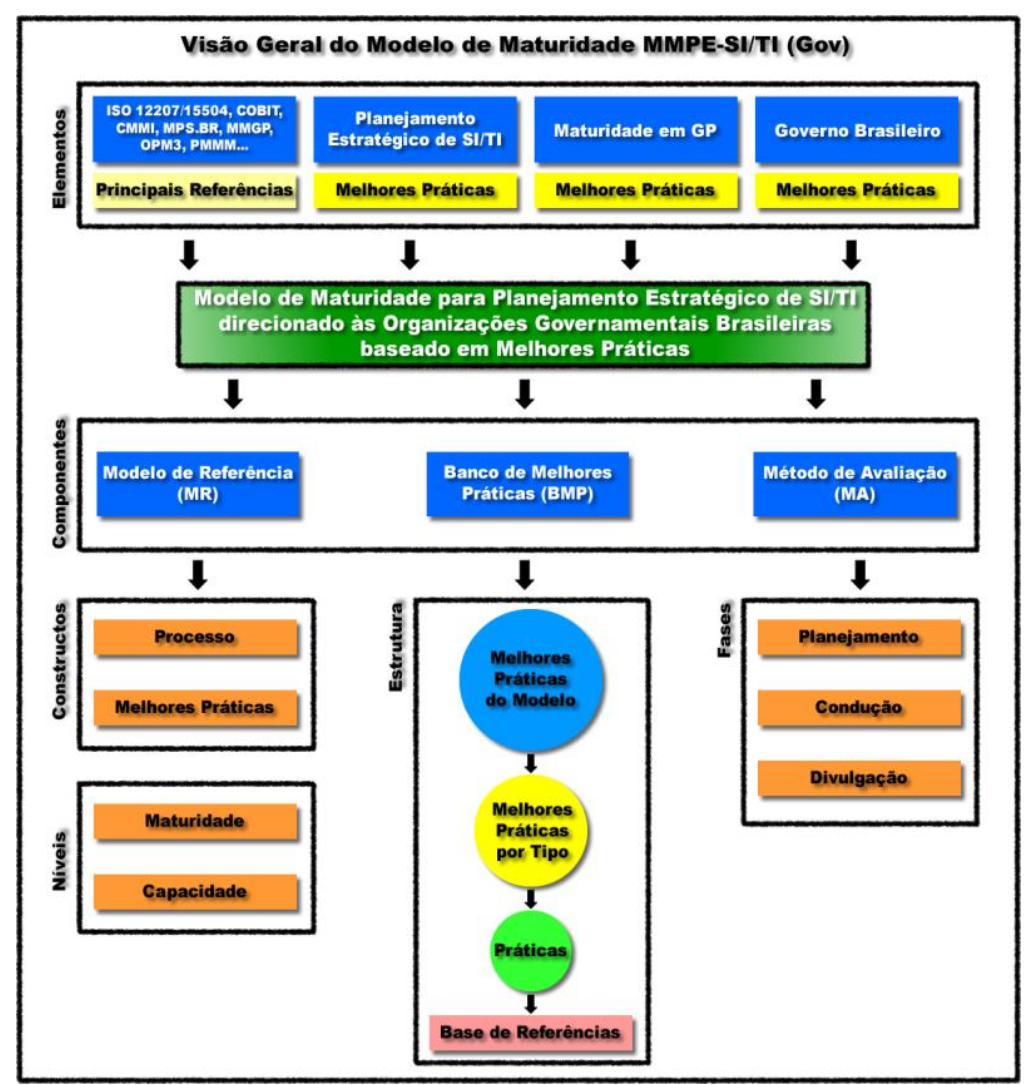

Figura 1: Visão geral do MMPE-SI/TI (Gov), [16]

O MMPE-SI/TI (Gov) possui 5 níveis de maturidade, 6 níveis de capacidade, 16 processos e 124 melhores práticas para planejamento estratégico de SI/TI direcionadas às organizações governamentais brasileiras (ver Figura 2).

\begin{tabular}{|c|c|c|c|}
\hline Nível & Processos & Áreas & Atributos do Processo \\
\hline 1 & $\begin{array}{l}\text { Promover Consciência Estratégica (PCE) } \\
\text { Assegurar Conformidade Governamental (ACG) }\end{array}$ & $\begin{array}{l}\text { Gestão } \\
\text { Organização }\end{array}$ & AP 1.1 \\
\hline 2 & $\begin{array}{l}\text { Gerenciar Recursos Humanos (GRH) } \\
\text { Educar e Treinar Pessoas (ETP) } \\
\text { Gerenciar Projetos (GEP) } \\
\text { Gerenciar Medição e Análise (GMA) }\end{array}$ & $\begin{array}{l}\text { Pessoas } \\
\text { Pessoas } \\
\text { Gestão } \\
\text { Gestão }\end{array}$ & AP $1.1 ;$ AP $2.1 ;$ AP $2.2 ;$ \\
\hline 3 & $\begin{array}{l}\text { Definir o Processo Organizacional (DPO) } \\
\text { Gerenciar Aquisições e Terceirizações (GAT) } \\
\text { Gerenciar Infraestrutura de SI/TI (GIN) } \\
\text { Gerenciar Qualidade (GQA) } \\
\text { Fomentar Gestão do Conhecimento (FGC) }\end{array}$ & $\begin{array}{l}\text { Organização } \\
\text { Organização } \\
\text { Tecnologia } \\
\text { Gestão } \\
\text { Organização }\end{array}$ & $\begin{array}{l}\text { AP } 1.1 ; \text { AP } 2.1 ; \text { AP } 2.2 ; \\
\text { AP } 3.1 ; \text { AP } 3.2 ;\end{array}$ \\
\hline 4 & $\begin{array}{l}\text { Avaliar o Processo Organizacional (APO) } \\
\text { Gerenciar Riscos (GRI) } \\
\text { Gerenciar Integração com o Cidadão (GIC) }\end{array}$ & $\begin{array}{l}\text { Organização } \\
\text { Gestão } \\
\text { Pessoas }\end{array}$ & $\begin{array}{l}\text { AP } 1.1 ; \text { AP } 2.1 ; \text { AP } 2.2 ; \\
\text { AP } 3.1 ; \text { AP } 3.2 ; \text { AP } 4.1 ; \\
\text { AP } 4.2 ;\end{array}$ \\
\hline 5 & $\begin{array}{l}\text { Melhorar o Processo Organizacional (MPO) } \\
\text { Otimizar a Gestão Organizacional (OGO) }\end{array}$ & $\begin{array}{l}\text { Organização } \\
\text { Gestão }\end{array}$ & $\begin{array}{l}\text { AP } 1.1 \text { AP } 2.1 ; \text { AP } 2.2 \text {; } \\
\text { AP } 3.1 ; \text { AP } 3.2 ; \text { AP } 4.1 ; \\
\text { AP } 4.2 ; \text { AP } 5.1 ; \text { AP } 5.2 ;\end{array}$ \\
\hline
\end{tabular}

Figura 2: Níveis de maturidade do modelo e seus processos, [16]

Este artigo tem como objetivo principal apresentar, em detalhes, um dos processos definidos pelo MMPESI/TI (Gov). O processo selecionado foi "Educar e Treinar Pessoas (ETP)", que contém um propósito, dois resultados esperados, sete melhores práticas e cinco produtos de trabalho.

Esse processo, que faz parte do Modelo de Referência (MR) do MMPE-SI/TI (Gov), foi definido com base em um extenso estudo fundamentado em revisão sistemática da literatura e encontra-se em conformidade 
com os principais modelos e normas nacionais e internacionais, como ISO/IEC 12207, MPS.BR: Guia Geral, COBIT, Art. $4^{\circ}$ do Decreto n ${ }^{\circ}$ 4.553, de 27 de dezembro de 2002 e Acórdão 1603/2008 TCU Plenário [45], [46], [23], [25], [28]. Esse processo, quanto ao seu propósito, resultados esperados e suas melhores práticas associadas, bem como os produtos de trabalho, será descrito a seguir.

Propósito: entender claramente as necessidades das pessoas (diretores, gerentes e usuários) em termos de educação e treinamento em SI/TI e executar uma estratégia eficaz de treinamento e medição dos resultados.

\section{Resultados esperados (RE):}

- ETP-RE-01: Treinamentos para tratar das necessidades da organização são desenvolvidos ou adquiridos;

- ETP-RE-02: Treinamentos para garantir que todos os indivíduos tenham habilidades necessárias para executar as suas tarefas são realizados, monitorados e avaliados.

\section{Melhores práticas (MP):}

- ETP-MP-01: Desenvolver uma estratégia de treinamento [RE: 1];

- $\quad$ ETP-MP-02: Identificar as necessidades de treinamento [RE: 1];

- ETP-MP-03: Desenvolver ou adquirir treinamento [RE: 1];

- ETP-MP-04: Preparar para executar o treinamento [RE: 1];

- $\quad$ ETP-MP-05: Realizar o treinamento do pessoal [RE: 2];

- ETP-MP-06: Manter os registros de treinamento [RE: 2];

- ETP-MP-07: Avaliar a eficácia do treinamento [RE: 2].

Produtos de trabalho (PT):

- $\quad$ ETP-PT-01: Estratégia de treinamento [RE: 1];

- $\quad$ ETP-PT-02: Plano de treinamento [RE: 1];

- ETP-PT-03: Material de treinamento [RE: 1];

- $\quad$ ETP-PT-04: Registro de treinamento [RE: 2];

- ETP-PT-05: Relatório de avaliação do treinamento [RE: 2].

Abaixo, observa-se uma lista completa das melhores práticas para o processo "Educar e Treinar Pessoas (ETP)". Cada melhor prática apresenta as seguintes informações: seu identificador (MP_ID), a nomenclatura utilizada (MP_NOME), a descrição da melhor prática (MP_DESCRIÇÃO) e a área a que a melhor prática está associada (MP_ÁREA) (ver Quadro 1). 
Quadro 1. Melhores práticas para educar e treinar pessoas (ETP), [16]

\begin{tabular}{|c|c|c|c|}
\hline MP_ID & MP_NOME & MP_DESCRIÇÃ̃O & MP_ÁREA \\
\hline ETP-MP-01 & $\begin{array}{l}\text { Desenvolver uma } \\
\text { estratégia de } \\
\text { treinamento. }\end{array}$ & $\begin{array}{l}\text { A organização deve criar uma estratégia para treinamento, incluindo a } \\
\text { forma como as necessidades de treinamento serão identificadas, como } \\
\text { os treinamentos serão desenvolvidos ou adquiridos e realizados. [RE: } \\
\text { 1]; }\end{array}$ & GES \\
\hline ETP-MP-02 & $\begin{array}{l}\text { Identificar as } \\
\text { necessidades de } \\
\text { treinamento. }\end{array}$ & $\begin{array}{l}\text { A organização deve identificar e avaliar habilidades e competências a } \\
\text { serem providenciadas ou melhoradas por meio de treinamentos. A } \\
\text { organização estabelece um plano de treinamento e atualiza, } \\
\text { regularmente, um currículo para cada grupo alvo de pessoas, } \\
\text { considerando: as estratégias, necessidades atuais e futuras do negócio; } \\
\text { o valor da informação como um bem; os valores corporativos (valores } \\
\text { éticos, culturais etc.); a implementação de uma nova infraestrutura de } \\
\text { SI/TI (pacotes e aplicações); as habilidades, as competências, as } \\
\text { certificações e atualizações necessárias; os métodos para ministrar as } \\
\text { aulas (em sala de aula, via web); o tamanho do grupo alvo, } \\
\text { acessibilidade e tempo. [RE: 1]; }\end{array}$ & ORG \\
\hline ETP-MP-03 & $\begin{array}{l}\text { Desenvolver ou } \\
\text { adquirir } \\
\text { treinamento. }\end{array}$ & $\begin{array}{l}\text { A organização deve desenvolver ou adquirir um treinamento que atenda } \\
\text { às necessidades comuns da organização e do pessoal de SI/TI. No caso } \\
\text { de treinamentos terceirizados, o contratante deve avaliar se está } \\
\text { cumprindo o que foi estabelecido em contrato e verificar, } \\
\text { periodicamente, se a contratada também está seguindo o que foi } \\
\text { acordado. [RE: } 1 \text { ]; }\end{array}$ & GES \\
\hline ETP-MP-04 & $\begin{array}{l}\text { Preparar para } \\
\text { executar o } \\
\text { treinamento. }\end{array}$ & $\begin{array}{l}\text { A organização deve identificar e preparar o portfólio de treinamentos, } \\
\text { incluindo a disponibilização de materiais para o treinamento e } \\
\text { disponibilização/liberação do pessoal que deve ser treinado, além de } \\
\text { publicar uma grade de treinamentos para os interessados. Com base nas } \\
\text { necessidades de treinamento identificadas, a organização define os } \\
\text { grupos alvo e seus membros, mecanismos adequados para ministrar os } \\
\text { treinamentos e selecionar os professores, instrutores e monitores para o } \\
\text { caso de ela mesma desenvolver seus treinamentos. Nesse caso, a } \\
\text { organização indica os instrutores e organiza os treinamentos da forma } \\
\text { mais adequada e oportuna à suas necessidades. [RE: 1]; }\end{array}$ & ORG \\
\hline ETP-MP-05 & $\begin{array}{l}\text { Realizar o } \\
\text { treinamento do } \\
\text { pessoal. }\end{array}$ & $\begin{array}{l}\text { A organização deve treinar o pessoal para ter o conhecimento e as } \\
\text { habilidades necessárias para desempenhar suas funções. [RE: 2]; }\end{array}$ & PES \\
\hline ETP-MP-06 & $\begin{array}{l}\text { Manter os } \\
\text { registros de } \\
\text { treinamento. }\end{array}$ & $\begin{array}{l}\text { A organização deve manter registros adequados dos treinamentos } \\
\text { realizados pelo pessoal de SI/TI. É fundamental registrar as inscrições } \\
\text { (incluindo pré-requisitos), a frequência de participação e realizar } \\
\text { avaliações de desempenho das pessoas que participaram do } \\
\text { treinamento. [RE: 2]; }\end{array}$ & TEC \\
\hline ETP-MP-07 & $\begin{array}{l}\text { Avaliar a eficácia } \\
\text { do treinamento. }\end{array}$ & $\begin{array}{l}\text { A organização deve identificar e avaliar o valor agregado por } \\
\text { treinamento desenvolvido ou adquirido, incluindo a avaliação do } \\
\text { material de treinamento. O treinamento recebido pode ser avaliado com } \\
\text { relação a conteúdo, relevância, qualidade, efetividade, absorção e } \\
\text { retenção do conhecimento, custo e valor. Os resultados dessa avaliação } \\
\text { devem servir de base para a definição dos futuros currículos e sessões } \\
\text { de treinamento. [RE: } 2 \text { ]. }\end{array}$ & GES \\
\hline
\end{tabular}

\section{Metodologia}

Para atender o objetivo central desta pesquisa, foram utilizados diversos procedimentos e técnicas apresentados a seguir:

Quanto ao método: as investigações, geralmente, utilizam uma combinação de dois ou mais métodos. Neste trabalho, adotaram-se os métodos indutivo, estruturalista e comparativo [36], [37].

Quanto à natureza: as pesquisas científicas podem ser classificadas em básica ou aplicada. Neste trabalho, foi utilizada a pesquisa aplicada, que objetiva gerar conhecimentos para aplicação prática e dirigida à solução de problemas específicos [38], [39].

Quanto aos procedimentos: foram realizados os seguintes procedimentos: análise documental, análise bibliográfica baseada em revisão sistemática da literatura, estudos de caso exploratórios, levantamento de campo (survey), estudo de campo baseado em Goal Question Metric (GQM) [40], [41], [36], [42], [43], [44].

Quanto às técnicas de coleta de dados: foram realizadas três entrevistas e aplicados questionários para auxiliar no processo de coleta de dados durante a realização dos estudos de caso exploratórios e durante a realização da validação do modelo junto aos especialistas da área de SI/TI [36], [37]. 
Quanto à abordagem: adotaram-se duas abordagens: quantitativa e qualitativa. Ambas serviram para analisar e discutir os resultados dos estudos de caso exploratórios e do levantamento realizado junto aos especialistas da área de SI/TI [36], [39].

Quanto à realização: utilizou-se o trabalho de campo como estratégia para atingir os objetivos pretendidos; neste caso, a pesquisa é realizada em um ambiente onde as variáveis não são controladas, o pesquisador se envolve no ambiente, observa e analisa as variáveis e características da realidade existente [36], [40].

Quanto à perspectiva: para Santos, a perspectiva representa o campo de interesse focado na pesquisa. Neste caso, foram considerados dois campos de pesquisa: Ciências da Computação e Ciências da Administração [39].

\section{Resultados}

Um conjunto de melhores práticas e o modelo de referência (MR) foram estabelecidos e validados junto aos especialistas da área de SI/TI, com base no GQM. As opiniões foram levantadas por meio de survey, utilizando questionários. Essas respostas foram analisadas e interpretadas a fim de demonstrar a importância, a capacidade, a confiabilidade e a coerência tanto das melhores práticas quanto do modelo de maturidade para planejamento estratégico de SI/TI, que também incluiu a validação dos propósitos e resultados esperados para cada um dos processos existentes no modelo, além de proporcionar as devidas correções e/ou melhorias propostas pelos especialistas.

A população ou o universo desta pesquisa foi composta por especialistas da área de SI/TI que trabalham, direta ou indiretamente, com planejamento estratégico de SI/TI em organizações públicas ou privadas, desde a sua elaboração/revisão/implementação até o desenvolvimento de pesquisas avançadas sobre o tema. Para este estudo, utilizou-se a amostragem não probabilística por conveniência [36], [37].

Os convites para os especialistas foram realizados exclusivamente por e-mail. O questionário foi enviado por e-mail e, também, por diversas redes sociais e grupos específicos (Linkedin, Twitter, Orkut, Facebook, Googlegroups, Yahoogroups) e esteve disponível no período compreendido entre 28/07 e 23/08/2010.

Um BMP e um MA também foram estabelecidos para o modelo e serão descritos a seguir.

\subsection{Banco de melhores práticas (BMP)}

O BMP definido para o MMPE-SI/TI (Gov) busca auxiliar as organizações governamentais brasileiras a atingirem um alto grau de maturidade em relação ao planejamento estratégico de SI/TI, por meio da utilização de um conjunto de melhores práticas.

Foi identificado, inicialmente, um conjunto de 505 práticas envolvendo as três áreas: planejamento estratégico de SI/TI, maturidade em gerenciamento de projetos e governo brasileiro. A partir desse conjunto inicial de práticas, foi estabelecido um conjunto de 80 MPs que representam as visões unificadas de diversas organizações e profissionais espalhados ao redor do mundo. Dessa reunião de MPs, $32(40 \%)$ são de planejamento estratégico de SI/TI, 30 (37\%) são de maturidade em gerenciamento de projetos e 18 (23\%) são específicas do governo brasileiro. Algumas foram divididas/desmembradas para garantir mais coerência e lógica contextual ao modelo.

Após esses ajustes, foi estabelecido o BMP para o modelo que contém um total de 124 melhores práticas para planejamento estratégico de SI/TI direcionado às organizações governamentais brasileiras. Desse total, 7 MPs estão associadas diretamente à área de educação e treinamento de pessoas, representada, nesse modelo, pelo processo "Educar e treinar pessoas (ETP)".

\subsection{Método de Avaliação (MA)}

Nota-se que as organizações precisam observar como funciona o método de avaliação (MA), ou seja, quais os requisitos necessários para se atingir determinado nível de maturidade/capacidade no modelo. 
Esse MA foi definido em conformidade com a norma internacional ISO/IEC 15504-1 [24] e referências como Kerzner [20], SCAMPI [27], OPM3 [21], MPS.BR: Guia de Avaliação [28] e ciclo PDCA [48].

Esse método combina três fases que juntas permitem proceder a uma avaliação completa da organização. Primeiramente, na fase de planejamento e preparação da avaliação, que é considerada a mais crucial do método, as pessoas envolvidas iniciam o levantamento das evidências (ex: dados, registros ou relatos de natureza qualitativa ou quantitativa) referentes à implementação de um elemento de processo a ser avaliado. A validação dessas evidências é realizada pela equipe de avaliação, para que sejam determinados os pontos fortes, fracos e as oportunidades de melhoria em relação ao modelo. Em seguida, os dados são documentados e submetidos a uma etapa de atribuição dos níveis de maturidade/capacidade para, finalmente, serem comunicados e arquivados (ver Figura 3).
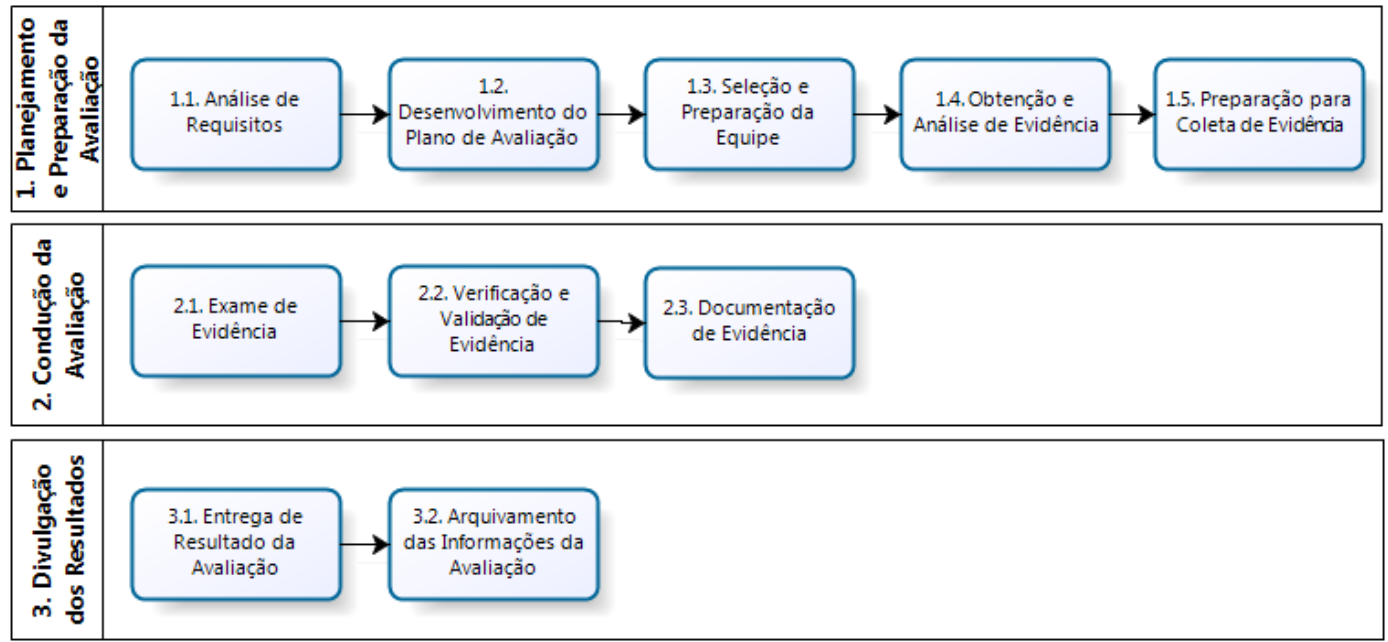

Figura 3: MA do MMPE-SI/TI (Gov), adaptado de [28], [27]

\subsection{Validação do processo "ETP"}

Para os especialistas de SI/TI, esse processo apresentou como maiores concentrações percentuais, em cada critério, os seguintes valores: totalmente importante $(54,4 \%)$, totalmente capaz $(39,6 \%)$, totalmente confiável $(39,3 \%)$ e totalmente coerente $(40,6 \%)$, conforme Gráfico 1.1. Diante dos resultados apresentados, pode-se constatar que, na média, 75,5\% dos respondentes consideraram esse processo como sendo "muito" e "totalmente" importante, capaz, confiável e coerente para todos os critérios.

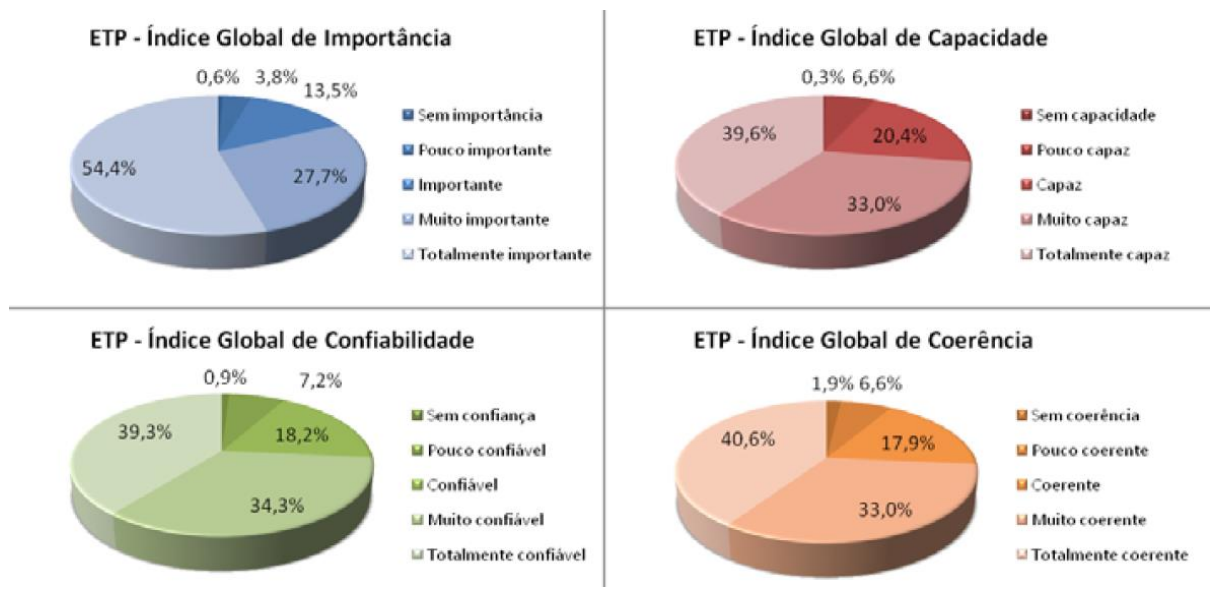

Gráfico 1.1. ETP: Índices globais de desempenho, [16] 
A seguir, são apresentadas as variáveis que melhor foram avaliadas pelos especialistas na categoria "totalmente" para os quatro critérios (ver Gráfico 1.2):

- ETP - propósito para o critério de "importância" com 59,4\% das opiniões, que trata de entender claramente as necessidades das pessoas (diretores, gerentes e usuários) em termos de educação e treinamento em SI/TI e executar uma estratégia eficaz de treinamento e medição dos resultados;

- ETP-RE-01 - para os critérios de "capacidade", "confiabilidade" e "coerência" com, respectivamente, $43,4 \%, 42,5 \%$ e $41,5 \%$ das opiniões, que aborda o desenvolvimento ou a aquisição de treinamentos para tratar das necessidades específicas da organização.

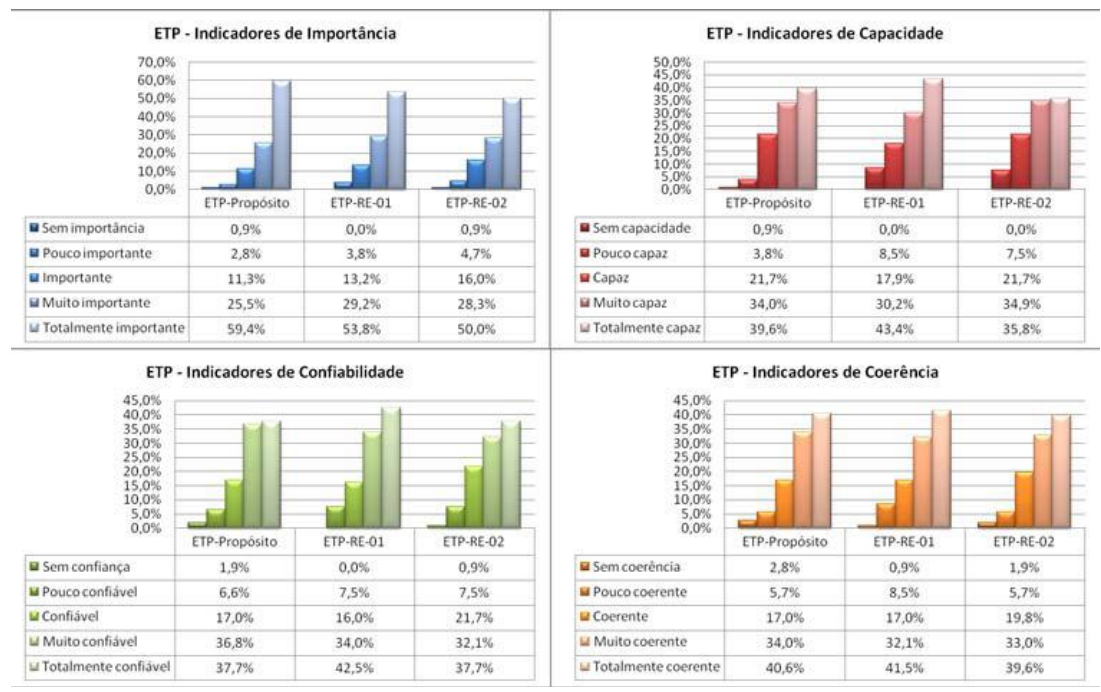

Gráfico 1.2. ETP: Indicadores de desempenho, [16] seguir:

Alguns dos comentários feitos pelos especialistas de SI/TI sobre esse processo são apresentados a

"Acho mais adequado falar em desenvolvimento de pessoas, e não em treinamento. Os processos de aprendizagem nas organizações não são apenas aqueles de capacitação formal, mas também de compartilhamento de experiência e aprendizado de atitudes, ambos aspectos importantes na área de TI." (E8).

"Falta: A contratante verifica o cumprimento dos compromissos de capacitação assumidos no contrato. Em muitos contratos, os colaboradores são forçados a assumir uma parte do custo (que deve ser custeado pela contratada) com treinamento sob a ameaça de ser substituído." (E13).

"Desenvolver ambientes amigáveis, vivenciais e, principalmente, de aplicação prática, com treinamento baseado em situações reais." (E54).

Em resposta ao comentário do especialista (E8), o modelo soluciona essa questão por meio da aplicação de gestão do conhecimento, que busca aumentar e melhorar o compartilhamento de informações e experiências entre as pessoas dentro e fora do ambiente. Algumas melhores práticas podem ser utilizadas com base no processo "Fomentar gestão do conhecimento (FGC)" situado no nível 3, sendo elas: "FGC-MP-02: Estabelecer um sistema de gestão do conhecimento", "FGC-MP-03: Criar uma rede de colaboradores do conhecimento" e "FGC-MP-05: Disseminar o conhecimento".

Com relação ao comentário do especialista (E13), o modelo realmente não tratava dessa situação; porém, a sugestão foi considerada interessante e foi aceita. Nesse caso, a melhor prática "ETP-MP-03" foi reformulada para contemplar a sugestão do especialista e poder responder completamente aos resultados esperados "ETP-RE-02", que, entre várias funções, já procurava avaliar o retorno do aprendizado, mas não observava se a organização (contratante) estava verificando e mantendo os compromissos de treinamento assumidos no contrato. 
O especialista (E54) comenta sobre a criação de treinamentos mais práticos que sejam baseados em situações reais. Nesse caso, cabe a cada organização governamental brasileira identificar suas necessidades e elaborar uma estratégia para determinar treinamentos mais adequados para responder melhor às suas exigências. A organização deve definir a infraestrutura necessária, os métodos que devem ser utilizados para ministrar as aulas (em sala de aula, via web) e se vai ela própria desenvolver os treinamentos ou se vai adquiri-los por meio de terceirização. $\mathrm{O}$ modelo sugere, para esse comentário em específico, que sejam implementadas várias melhores práticas, entre elas: "ETP-MP-01: Desenvolver uma estratégia de treinamento", "ETP-MP-02: Identificar as necessidades de treinamento" e "ETP-MP-03: Desenvolver ou adquirir treinamento" [47], [45], [26], [25], [30], [32], [20], [21], [6].

\subsection{Apoio para a implementação do processo ETP}

De acordo com o propósito do processo ETP, foi possível identificar cinco ferramentas e técnicas na literatura que podem ser utilizadas para apoiar sua implementação. Para tal, foram definidas sete categorias relacionadas às características e funcionalidades dessas ferramentas e técnicas, e cada uma delas foi comparada de acordo com essas categorias. As ferramentas/técnicas selecionadas foram Balanced ScoreCard, Mapa Estratégico, Análise SWOT, Reuniões e Workshops e ProManager (ver Quadro 2).

Observa-se, no Quadro 2 que a ferramenta ProManager oferece a maior quantidade de características/funcionalidades categorizadas para apoiar o processo ETP, em comparação às demais ferramentas e técnicas, quatro no total.

Quadro 2: Características das ferramentas e técnicas para apoiar o processo ETP

\begin{tabular}{|l|c|c|c|c|c|}
\hline \multicolumn{1}{|c|}{ Categorias } & $\begin{array}{c}\text { Balanced } \\
\text { ScoreCard }\end{array}$ & $\begin{array}{c}\text { Mapa } \\
\text { Estratégico }\end{array}$ & $\begin{array}{c}\text { Análise } \\
\text { SWOT }\end{array}$ & $\begin{array}{c}\text { Reuniões e } \\
\text { Workshops }\end{array}$ & ProManager \\
\hline Apoio na tomada de decisão & & & & & $\checkmark$ \\
\hline $\begin{array}{l}\text { Coletar opiniões/discussão de } \\
\text { soluções }\end{array}$ & $\checkmark$ & & $\checkmark$ & & $\checkmark$ \\
\hline $\begin{array}{l}\text { Planejamento e controle das } \\
\text { atividades/tarefas }\end{array}$ & & & & & $\checkmark$ \\
\hline $\begin{array}{l}\text { Verificar a capacidade da } \\
\text { organização }\end{array}$ & $\checkmark$ & $\checkmark$ & $\checkmark$ & & $\checkmark$ \\
\hline $\begin{array}{l}\text { Avaliação/Feedback do } \\
\text { desempenho }\end{array}$ & $\checkmark$ & & & & \\
\hline $\begin{array}{l}\text { Posição da organização no } \\
\text { mercado }\end{array}$ & & $\checkmark$ & & $\checkmark$ & \\
\hline $\begin{array}{l}\text { Facilitar/Melhorar a comunicação } \\
\text { organizacional }\end{array}$ & & & & & \\
\hline
\end{tabular}

\section{Conclusão}

Durante este estudo, foi possível verificar a relevância do planejamento estratégico de SI/TI para as organizações governamentais brasileiras. Aspectos como solidez da estratégia de negócio, avaliação da qualidade e dos resultados e estudos e ações para tratar as vulnerabilidades associadas aos riscos podem não ser realizados sem a utilização desse planejamento.

Este artigo apresentou o processo ETP, que faz parte do modelo MMPE-SI/TI (Gov), criado como resultado de uma tese de doutorado [16]. Esse processo tem o objetivo de compreender o que é essencial aos integrantes de uma organização, com relação à educação e ao preparo relacionado ao SI/TI e à utilização de uma estratégia para preparar e mensurar os resultados. Uma lista de resultados foi gerada descrevendo os resultados esperados para a obtenção de um desempenho positivo desse processo. Os resultados esperados estabelecem o que se almeja obter com a efetiva implementação do processo, podendo ser evidenciados por um produto de trabalho produzido ou uma mudança significativa de estado, ao se executar o processo.

Foram descritas, também, as melhores práticas que devem ser implementadas para gerar esses resultados esperados. Essas melhores práticas foram obtidas por meio de um levantamento exaustivo e, depois, adaptadas para o contexto das organizações governamentais brasileiras. A organização deve utilizar as melhores 
práticas para atingir o propósito desse processo, o que permite avaliar e atribuir graus de efetividade na sua execução.

Na média, 75,5\% dos respondentes afirmou que o processo ETP era "muito" e "totalmente" importante, capaz, confiável e coerente em todos os critérios. Essa informação demonstra o quanto esse processo é importante para instruir e preparar as pessoas em uma organização governamental brasileira, como também foi considerado amplamente capaz de ser implementado.

As ferramentas e técnicas descritas neste artigo não devem ser percebidas como as únicas a serem usadas na implementação do processo ETP, mas sim encaradas como sugestões que podem propiciar uma melhor educação e um melhor treinamento dos indivíduos. Cada organização pode utilizar/identificar as ferramentas e técnicas mais interessantes para seu contexto, e as que foram citadas servem, principalmente, como um guia para dar suporte às organizações governamentais brasileiras.

\section{Referências}

[1] REZENDE, D. A. Planejamento de Sistemas de Informação e Informática: guia prático para planejar a Tecnologia da Informação integrada ao planejamento estratégico das organizações. 2. ed. São Paulo: Atlas, 2007.

[2] BRASIL. Tribunal de Contas da União. Levantamento acerca da Governança de Tecnologia da Informação na Administração Pública Federal. Relator Ministro Benjamin Zymler. Brasília: TCU, SEFTI - Secretaria de Fiscalização de Tecnologia da Informação, 2008.

[3] BOYNTON, A. C.; ZMUD, R. W. Information technology planning in the 1990's: directions for practice and research. MIS Quarterly, v. 11, p. 59-71, 1987.

[4] EARL, M. J. Management Strategies for Information Technology. London: Prentice Hall International, 1989.

[5] EARL, M. J. Experiences in strategic information systems planning. MIS Quarterly, v. 17, p. 1-24, 1993.

[6] SEGARS, A. H.; GROVER, V. Strategic Information Systems Planning Success: an investigation of the construct and its measurements. MIS Quarterly, v. 22, n. 2, p. 139-163, 1998.

[7] FINNEGAN, P. et al. Applying triple loop learning to planning electronic trading systems. Information Technology \& People, v. 16, n. 4, p. 461-483, 2003.

[8] APPLEBY, T. et al. Advancing Organizational Project Management Maturity. Havertown, USA: CBP Center for Business Practices, 2007.

[9] CRAWFORD, J. K. Project Management Maturity Model (CBP - Center for Business Practices). 2. ed. Boca Raton, USA: Auerbach Publications, 2007.

[10]-OFFICE OF GOVERNMENT COMMERCE (OGC). Portfolio, Programme and Project Management Maturity Model (P3M3). Versão 2.0. London: OGC, 2008.

[11]-OFFICE OF GOVERNMENT COMMERCE (OGC). PRINCE2 Maturity Model (P2MM). Versão 1.0. London: OGC, 2006.

[12] PENNYPACKER, J. S. Project Portfolio Management Maturity Model. Havertown, USA: CBP - Center for Business Practices, 2005.

[13] LI, M.; YE, L. R. Information Technology and Firm Performance: linking with environmental, strategic and managerial contexts. Information \& Management, v. 35, p. 43-51, 1999.

[14] PEREZ, B. E. Information Systems Investment Decisions in Business Practice: the spanish case. European Journal of Information Systems, v. 7, p. 202-209, 1998.

[15] THONG, J. Y. L. An Integrated Model of Information Systems Adoption in Small Business. Journal of Management Information Systems, v. 15, n. 4, p. 187-214, 1999.

[16] TEIXEIRA FILHO, J. G. A. MMPE-SI/TI (Gov) - Modelo de Maturidade para Planejamento Estratégico de SI/TI direcionado às Organizações Governamentais Brasileiras baseado em Melhores Práticas. vols. 1 e

Revista Brasileira de Computação Aplicada (ISSN 2176-6649), Passo Fundo, v. 5, n. 2, p. 28-39, out. 201337 
2, 2010. Tese (Doutorado em Ciências da Computação) - Universidade Federal de Pernambuco (UFPE), Recife, 2010.

[17]- PROJECT MANAGEMENT INSTITUTE (PMI). How Mature is Your Organization - Really?. PM Network, v. 23. n. 2, 2009. ISSN 1040-8754.

[18] LAUDON, K. C.; LAUDON, J. P. Sistemas de Informação Gerenciais. 7. ed. São Paulo: Pearson Prentice Hall, 2007.

[19]-CENTER OF BUSINESS PRACTICES (CBP). Project Management Maturity: a benchmark of current best practices. West Chester, USA, 2006. Disponível em: 〈http://www.cbponline.com/>. Acesso em: 10 jul. 2007.

[20] KERZNER, H. Using the Project Management Maturity Model: strategic planning for project management. 2. ed. Hoboken, USA: John Wiley \& Sons, 2005.

[21]-PROJECT MANAGEMENT INSTITUTE (PMI). Organization Project Management Maturity Model (OPM3). Newton Square: PMI, 2003.

[22] KERZNER, H. Project Management Best Practices: achieving global excellence. Hoboken, USA: John Wiley \& Sons, 2006.

[23] ISO/IEC 12207. International Organization for Standardization/International Electrotechnical Comission. Systems and Software Engineering - Software Life Cycle Processes. Geneve: ISO, 2008.

[24] ISO/IEC 15504-1. International Organization for Standardization/International Electrotechnical Comission. Information Technology - Process Assessment - Part 1: concepts and vocabulary. Geneve: ISO, 2004.

[25] ITGI - IT Governance Institute. COBIT - Framework Control Objectives Management Guidelines Maturity Models. Versão.1. USA: ITGI, 2007.

[26] PRADO, D. S. Maturidade em gerenciamento de projetos. Nova Lima: INDG Tecnologia e Serviços Ltda., 2008.

[27] SOFTWARE ENGINEERING INSTITUTE (SEI). -CMMI for Development: improving processes for better products. CMMI-DEV 1.2. Pittsburgh, PA: Carnegie Mellon University, 2006.

[28]ASSOCIAÇÃO PARA PROMOÇÃO DA EXCELÊNCIA DO SOFTWARE BRASILEIRO (SOFTEX). MPS.BR - Guia Geral. 2009. Disponível em: <http://www.softex.br/>. Acesso em: 09 dez. 2010.

[29] TEUBNER, R. A. Strategic Information Systems Planning: a case study from the financial services industry. Journal of Strategic Information Systems, v. 16, p. 105-125, 2007.

[30] GORDON, S. R.; GORDON, J. R. Sistemas de Informação: uma abordagem gerencial. 3. ed. Rio de Janeiro: LTC, 2006.

[31] NEWKIRK, H. E.; LEDERER, A. L. The effectiveness of strategic information systems planning under environmental uncertainty. Information \& Management, v. 43, p. 481-501, 2006.

[32] CASSIDY, A. A Pratical Guide to Information Systems Strategic Planning. 2. ed. Boca Raton, USA: Auerbach Publications, 2005.

[33] LAUDON, K. C.; LAUDON, J. P. Sistemas de Informação Gerenciais: administrando a empresa digital. 5. ed. São Paulo: Pearson Prentice Hall, 2004.

[34] O'BRIEN, J. A. Sistemas de Informação e as decisões gerenciais na era da Internet. 2. ed. São Paulo: Saraiva, 2004.

[35] SALMELA, H. et al. Information Systems Planning in a Turbulent Environment. European Journal of Information Systems, v. 9, n. 1, p. 3-15, 2000.

[36] GIL, A. C. Métodos e técnicas de pesquisa social. 6. ed. São Paulo: Atlas, 2009.

[37] LAKATOS, E. M.; MARCONI, M. A. Metodologia científica. 5. ed. São Paulo: Atlas, 2009.

[38] CERVO, A. L. et al. Metodologia científica. 6. ed. São Paulo: Pearson Prentice Hall, 2007.

Revista Brasileira de Computação Aplicada (ISSN 2176-6649), Passo Fundo, v. 5, n. 2, p. 28-39, out. 201338 
[39] SANTOS, A. R. Metodologia científica: a construção do conhecimento. 5. ed. Rio de Janeiro: DP\&A, 2002.

[40] BIOLCHINI, J. et al. Systematic Review in Software Engineering: relevance and utility. Relatório Técnico RT-ES-679/05, Programa de Engenharia de Sistemas e Computação (PESC), COPPE/UFRJ, 2005.

[41] DYBA, T.; KAMPENES, V.; SJOBERG, D. A Systematic Review of Statistical Power in Software Engineering Experiments. Journal of Information and Software Technology, v. 1, n. 11, 2005.

[42] KITCHENHAM, B. A.; DYBA, T.; JORGENSEN, M. Evidence-based Software Engineering. Proceedings of the 26th International Conference on Software Engineering (ICSE'04), p. 273-281, 2004.

[43] LITTELL J. H.; CORCORAN J.; PILLAI, V. Systematic Reviews and Meta-analysis. Oxford, USA: Oxford University Press, 2008.

[44] MAFRA, S. N.; TRAVASSOS, G. H. Estudos primários e secundários apoiando a busca por evidência em Engenharia de Software. Relatório Técnico RT-ES-687/06, Programa de Engenharia de Sistemas e Computação (PESC), COPPE/UFRJ, 2006.

[45] BRASIL. Acórdão 1603/2008 TCU Plenário. 2008. Disponível em: <www.mp.go.gov.br/portalweb/hp/12/docs/acordao_tcu_-_13-08-2008.pdf>. Acesso em: 20 jan. 2009.

[46] BRASIL. Decreto $\mathrm{n}^{\circ} 4.553$, de 27 de dezembro de 2002 . Disponível em: <http://www.planalto.gov.br/CCIVIL/decreto/2002/D4553.htm>. Acesso em: 25 jan. 2009.

[47] BRASIL. Lei $\mathrm{n}^{\mathrm{o}} 11.907$, de 2 de fevereiro de 2009. Disponível em: <https://www.planalto.gov.br/ccivil_03/_Ato2007-2010/2009/Lei/L11907.htm>. Acesso em: 22 jul. 2009.

[48] CAMPOS, V. F. TQC - Controle da Qualidade Total. Nova Lima: INDG Tecnologia e Serviços Ltda., 2004. 